\title{
The Absence of X-ray Flashes from Nearby Galaxies and the Gamma-Ray Burst Distance Scale
}

\author{
T. T. Hamilton \\ Department of Astronomy \\ California Institute of Technology, Pasadena, CA 91125 \\ Electronic Mail: tth@astro.caltech.edu \\ E. V. Gotthelf ${ }^{1}$ \\ Laboratory for High Energy Astrophysics \\ NASA/Goddard Space Flight Center, Greenbelt, MD 20771 \\ Electronic Mail: gotthelf@gsfc.nasa.gov \\ and \\ D. J. Helfand \\ Department of Astronomy and Columbia Astrophysics Laboratory \\ Columbia University, 538 West 120th Street, New York, NY 10027 \\ Electronic Mail: djh@carmen.phys.columbia.edu
}

To appear in the Astrophysical Journal

${ }^{1}$ Universities Space Research Association 


\begin{abstract}
If typical gamma-ray bursts (GRBs) have X-ray counterparts similar to those detected by Ginga, then sensitive focusing X-ray telescopes will be able to detect GRBs three orders of magnitude fainter than the detection limit of the Burst and Transient Source Experiment (BATSE). If a substantial portion of the burst population detected by BATSE originates in a Galactic halo at distances greater than or equal to $150 \mathrm{kpc}$, existing X-ray telescopes will be able to detect GRBs in external galaxies out to a distance of at least 4.5 Mpc. As reported in Gotthelf, Hamilton, \& Helfand (1996) the Imaging Proportional counter (IPC) on board the Einstein Observatory detected 42 transient events with pointlike spatial characteristics and timescales of less than 10 seconds. These events are distributed isotropically on the sky; in particular, they are not concentrated in the directions of nearby external galaxies. For halo models of the BATSE bursts with radii of $150 \mathrm{kpc}$ or greater, we would expect to see several burst events in observations pointed towards nearby galaxies. We see none. We therefore conclude that if the Ginga detections are representative of the population of GRBs sampled by BATSE, GRBs cannot originate in a Galactic halo population with limiting radii between 150 $\mathrm{kpc}$ and $400 \mathrm{kpc}$. Inasmuch as halos with limiting radii outside of this range have been excluded by the BATSE isotropy measurements, our result indicates that all halo models are excluded. This result is independent of whether the flashes we do detect have an astronomical origin.
\end{abstract}

Subject headings: gamma rays: bursts - surveys - X-rays: bursts 


\section{Introduction}

Although their existence has been recognized for over two decades, gamma-ray bursts (GRBs) remain enigmatic, their distances and inherent luminosities uncertain by many orders of magnitude. In recent years our understanding has increased enormously as a consequence of the isotropy and apparent luminosity-function measurements carried out by the BATSE instrument onboard the Compton Gamma-Ray Observatory (CGRO) (Meegan et al. 1992; Hakkila et al. 1994a, 1994b; see Fishman et al. 1989 for a discussion of the BATSE experiment). The preponderance of evidence suggests that GRBs originate at one of two possible classes of sites, either in an extended Galactic halo or at cosmological redshifts. Many workers have developed models in which the GRBs arise from a halo population at distances of tens to hundreds of kiloparsecs from the Galactic Center (e.g., Smith \& Lamb 1993; Podsiadlowski, Rees, \& Ruderman 1995). In this scenario, the observed inhomogeneity in the number-size relation is understood as the result of the finite extent of the halo. The BATSE results have effectively excluded models with limiting halo radii of less than $150 \mathrm{kpc}$ (Hakkila et al. 1994a). Other workers have proposed that GRBs originate at cosmological distances. The inhomogeneity is then understood as a result of a combination of evolutionary effects and redshift-induced spectral effects (Paczynski et al. 1986; Paczynski \& Rhoads 1993 and references therein).

In this paper we propose and execute a new test of Galactic halo models. We begin with a review of the observed X-ray properties of GRBs and outline our strategy for using existing X-ray imaging data to constrain models of the GRB source distribution $(\S 2)$. We then define the halo models and construct a catalog of nearby galaxies whose halos were observed by the Einstein IPC. Section 4 presents our principal result - the complete absence of bursts from nearby galaxies - and uses this to constrain burst distances. The final section examines the robustness of our conclusions and summarizes our results.

\section{X-Rays from GRBs}

If our Galaxy is typical, Galactic halo models predict that external galaxies will have sources of GRBs similar to those surrounding the Milky Way, presumably with similar spectral and temporal characteristics. GRBs in these external halos would, of course, be much fainter than those from the halo of our Galaxy. Because absorption effects will be insignificant over the distances to nearby galaxies, however, measurement of the flux of GRBs from the halo of an external galaxy at a known distance would provide an immediate measure of the intrinsic burst luminosity and, hence, the distance of Galactic GRBs. Similarly, an upper limit on the flux of GRBs in nearby galaxies provides, in the context of halo models, a lower limit on the distance of the BATSE detected bursts.

If we assume that the faintest BATSE bursts originate at a distance of $150 \mathrm{kpc}$, the smallest limiting distance consistent with the BATSE isotropy tests, it is clear that bursts from galaxies well beyond M31 will be fainter than the BATSE limit by orders of magnitude. The only instruments that have any chance of detecting high energy sources at such faint flux levels are focusing X-ray telescopes such as those carried by Einstein, ROSAT and the Advanced Satellite for Cosmology and Astrophysics (ASCA). Unfortunately, such telescopes are confined to low energy bands at which BATSE spectroscopy is non-existent. We have therefore assumed for the present experiment that typical GRBs have X-ray spectra similar to the spectra of the GRBs observed by Ginga (see below). The two largest available databases of X-ray observations are from the Einstein and ROSAT position sensitive proportional counters. To minimize the uncertainty introduced by the requisite extrapolation from the Ginga 1.5 to $10 \mathrm{keV}$ band, we analyze Einstein data in preference to the somewhat softer photons recorded in the ROSAT database. Nevertheless, the typical X-ray flux associated with GRBs is highly uncertain and remains the greatest source of uncertainty in our experiment. We discuss this in more detail in section 4.

Ginga observed a total of 17 GRBs with a mean flux in the $1.5-10 \mathrm{keV}$ band of $\sim 4 \%$ that of the gamma-ray flux (Yoshida et al. 1993). Spectral analysis of the brightest of these bursts showed a thermal spectrum with a best fit bremsstrahlung temperature of $1.5 \mathrm{keV}$ (Murakami et al. 1990). While many papers presenting Ginga results interpreted them in terms of a blackbody spectral model, this was motivated by the coincidence that the observed Ginga burst flux equaled the flux expected from a blackbody with the classical neutron star radius of $10 \mathrm{~km}$ at a distance of $1 \mathrm{kpc}$. The observed Ginga spectrum is consistent with that of a $1.5 \mathrm{keV}$ thermal bremsstrahlung continuum. We adopt this model 
here, not because we consider it to have any physical significance, but because it is a convenient parameterization of the best data available on GRB X-ray spectra.

The XMON experiment aboard P78-1 also detected $3-10 \mathrm{keV}$ X-ray counterparts to GRBs, and found flux ratios similar to those detected by Ginga (Laros et al. 1984). Both the Ginga and XMON results indicate X-ray fluxes somewhat higher than a naive extrapolation of the burst power law spectrum observed between 40 and $70 \mathrm{keV}$ (from the composite of all BATSE bursts - Band et al. 1993). Inasmuch as Ginga was only sensitive to the hard portion of this thermal excess, it is possible that the effective temperature is less than the Ginga fits with $k T=1.5 \mathrm{keV}$. In that case, the X-ray emission in the Einstein IPC band would be greater than we assume here.

No previous experiment has detected absorption of $\mathrm{X}$-rays by material either local to the burst source or lying along the line of sight. However, since our working band is softer, significant absorption could affect Einstein-observed bursts. Because most of the galaxies we include in our sample lie in regions of the sky in which Galactic absorption is only $\approx 10^{20} / \mathrm{cm}^{-2}$ (Stark et al. 1992), such line-of-sight absorption is not a major issue. However absorption local to the burst emitter with a column density in the range $10^{21}$ to $10^{23} \mathrm{~cm}^{-2}$ could greatly reduce the source fluence in the IPC band. Such absorbers would have to be physically large (and at least several AU away from the burst) however, in order that the flux from the burst would not fully ionize the absorber, allowing $\mathrm{X}$-rays through.

The composite Ginga GRB X-ray spectrum, folded through the Einstein spectral response function using the PIMMS software, yields an expected count rate in the Einstein IPC of 540 counts per second for a burst with a flux of $2 \times 10^{-7} \mathrm{erg} \mathrm{cm}^{-2}$ in the BATSE band, the limit to which the BATSE team has calculated a reliable number-size relation. Since the background count rate in the IPC is almost always less than one count per thousand seconds per resolution element, an event of a few counts in a ten-second interval stands out dramatically and can easily be detected (Gotthelf, Hamilton, \& Helfand 1996, hereafter Paper I). We therefore are sensitive to GRBs out to a distance 30 times greater than the distance of the faintest BATSE bursts.

Both BATSE and our experiment are flux-limited. The longest timescale on which BATSE triggers to record a burst is $1024 \mathrm{~ms}$, and burst durations range from tens of milliseconds to hundreds of seconds. We are sensitive to X-ray events primarily on a timescale of 1 to 10 seconds and have defined the flux limit of our survey accordingly. If the X-ray bursts are longer than 10 seconds, this is a conservative approach, but if the X-ray counterparts of GRBs frequently had timescales much shorter than $10 \mathrm{~s}$, our flux sensitivity will be proportionately lower than we have estimated. We note that all observed X-ray counterparts of GRBs in fact indicate longer timescales for the X-ray emission; indeed, some of the burst we detect have tails extending to $100 \mathrm{~s}$ (see figure 1 of Paper I). At higher energies, investigators have also noticed a correlation between softer bursts and longer timescales (Yoshida et al. 1989; Laros et al. 1984; Norris et al. 1986). While these results are not conclusive, we consider that, since no observation of a X-ray counterpart to a GRB has detected such an event with a timescale shorter than 10 seconds, we are justified in our sensitivity calculation.

It is possible that events other than GRBs could also produce X-ray flashes in the IPC (Paper I). However, it is not necessary that we understand possible alternative sources of transients in order to test the definite prediction that halo models make regarding extragalactic GRB X-ray counterparts.

\section{Halo Models and the Catalog of Galaxies Observed}

\subsection{Halo Models}

The principal interest in the absence of X-ray transients from nearby galaxies is the significance of this non-detection as a test of halo models of GRB sites. The crucial question here is the number of bursts that we would expect to detect if GRBs do originate in the Galaxy's halo. This depends of course on the number of bursts in our Galactic halo and their intrinsic luminosity. We here make the extremely conservative assumption that their are no GRBs fainter than those observed by BATSE. We adopt a rate for the Milky Way of 1500 bursts per year. We derive this number from the efficiency calculations of the BATSE team who estimate that BATSE is sensitive to about one quarter of the bursts occurring at the faintest flux levels (Meegan et al. 1994), and apply an additional correction factor of 1.28 to account for our position off-center in the Galactic halo.

The number of bursts expected to be detected by 
Einstein is a sensitive function of the limiting halo radius in two ways. If the BATSE bursts come from a larger halo, then they are intrinsically more luminous and their X-ray counterparts could thus be detected from more distant galaxies. On the other hand, a more extended halo means that the surface density of bursts in external galaxies will be lower. As a practical matter, given the existence of a limited set of observations in the Einstein database, these two effects work against each other. If bursts originate in relatively extended halos, then the number of bursts per unit surface area per unit time will be less for individual galaxies. However, a relatively extended halo implies a relatively high intrinsic burst luminosity. Therefore they can be seen at greater distances and more existing Einstein fields would be expected to contain bursts.

We have calculated the expected number of bursts for all halo models consistent with the BATSE isotropy result. The adopted lower limit for the limiting burst distance of $150 \mathrm{kpc}$ follows from the upper limit on the GRB quadrapole moment with respect to the plane of the Galaxy, and the upper limit of $400 \mathrm{kpc}$ follows from the upper limit to the dipole with respect to M31 (Hakkila et al. 1994b). We calculate the expected surface density of bursts $\rho_{s}$ at a distance $r$ from the center of the Galaxy for burst source models in which

$$
\begin{array}{cc}
\rho_{s} \propto \frac{1}{1+\left(r / r_{c}\right)^{\alpha}}, & r<r_{l i m}, \\
\rho_{s}=0, & r>r_{l i m},
\end{array}
$$

where $\alpha \approx 2$, and the population abruptly cuts off at a radius $r_{l i m}$. This formalism is commonly used in the analysis of BATSE data, primarily because it is similar to models of dark matter distributions that are invoked to explain galaxy velocity profiles (Fich \& Tremaine 1991; Innanen, Harris, \& Webbink 1983). Models in which $\alpha<2$ can also fit the BATSE data and may be physically more reasonable; as shown in Hakkila et al. (1994a), such models require a larger limiting radius. We adopt the model with the conservative assumption that $\alpha=2$, not because of any belief in its physical significance, but because the use of such a model facilitates interpretation of our results in the context of other GRB studies, especially those interpreting BATSE data.

The surface density in such models is not significantly dependent on the value of $r_{c}$, the softening parameter in the burst site distribution. For all values of $r_{c}$ substantially less than $r_{\text {lim }}$, the expected projected surface density of sources at the center of the halo is $\rho_{s}=6.9\left(D / D_{\text {lim }}\right)^{2}$ per $10^{6}$ seconds $\mathrm{deg}^{-2}$, where $D_{\text {lim }}$ is the maximum distance to which a BATSE burst at $r_{l i m}$ could be detected. For a halo limit of $150 \mathrm{kpc}$ implying $D_{\text {lim }}=4.5 \mathrm{Mpc}$, we expect one burst every 145,000 seconds in the Einstein field of view. A $150 \mathrm{kpc}$ halo at $4.5 \mathrm{Mpc}$ subtends roughly $10 \operatorname{deg}^{2}$ as indeed does any halo of size $r_{l i m}$ viewed at the distance corresponding to the limiting sensitivity.

\subsection{The Galaxy Catalog}

A complete list of nearby galaxies with distances from 1 to $12 \mathrm{Mpc}$ and with $M_{v}<-16$ was drawn from the Nearby Galaxies Atlas (Tully 1989). We have excluded all galaxies that are within 30 arcmin of a brighter galaxy at the same distance in order to ensure that satellite galaxies deep within the halo of a larger galaxy are not counted as independent objects. We have not applied any weighting by mass to the galaxies. In our calculations we have formally assumed that the typical galaxy we observe has a halo identical to that of the Milky Way. The observation times are skewed somewhat towards more luminous galaxies, which were more likely to be chosen as IPC targets for reasons unrelated to our search. This means that assuming all catalog galaxies to be equal contributors to the burst population is a conservative assumption with regard to the distribution of bursts. If we weighted the galaxies, any plausible scheme would place more weight on the systems which were in fact most observed.

This does not, however, resolve the question of the overall normalization of the total galaxy luminosity in our sample. Gott \& Turner (1976) estimate that the local density of galaxy optical luminosity is about 2.75 times the optical luminosity density on large scales. Adopting their numbers, we calculate that our assumptions are equivalent to assuming that the burst/galaxy luminosity ratio for our sample is approximately 1.6 times the value for the Galaxy. Specifically, we assume that the total burst-producing material along the line of sight to our sample galaxies has a ratio to those galaxies' luminosity 1.6 times as great as the ratio of burst-producing material within the model radius of the Milky Way to our Galaxy's luminosity. This is roughly comparable to assuming that burst production traces mass and applying standard comparisons of mass to light ratios for galaxies. If a substantial fraction of the intergalactic mass in- 
ferred from kinematic studies emits bursts, then the expected bursts will be correspondingly more numerous. Trimble (1987) provides a thorough review of the uncertainties of computing galactic and intergalactic masses in regions with no visible emission. The fact that the mass of material far from the luminous regions of the disk is so uncertain leads us to our simple approach.

We next constructed a database containing all IPC pointings whose centers lay within 5 degrees of any of the 189 galaxies in our catalog, thus including both observations that were deliberately pointed at a nearby galaxy and serendipitous observations in which a galaxy or part of its putative halo is within the field of view. A total of $2.8 \times 10^{6}$ seconds was accumulated, with most of the time spent in scheduled observations of well-known nearby galaxies; one flash was detected. Since Einstein detected 18 potentially astronomical flashes in $1.6 \times 10^{7}$ seconds this is not statistically unexpected. This result is not dependent on the arguments used in Paper I to extract the 18 potentially astronomical events from the complete list of 42 candidates; none of the 24 likely counter events fell within the nearby galaxy database. Table 1 lists the galaxy positions, distances and the total time that Einstein spent observing a putative 400 kpc halo about each galaxy's position. The observing times for nearby galaxies are large. However, as explained above, the expected surface density of bursts is low for nearby galaxies, and, as a result, most of the contribution to the expected burst total comes from galaxies near the limiting distance for a particular halo model.

Since larger halo models imply higher luminosities for the BATSE burst sample, we must examine observations of galaxies at larger distances as the assumed $r_{\text {lim }}$ increases. A $200 \mathrm{kpc}$ radius halo would produce bursts visible out to $6 \mathrm{Mpc}$, while a $400 \mathrm{kpc}$ halo is visible to $12 \mathrm{Mpc}$ and so on. Similarly, the surface density of bursts from the halos of galaxies at distances less than that of the limiting sensitivity is reduced by a factor proportional to the square of the ratio of the distance to the limiting distance.

\subsection{Results}

Table 2 lists the predicted number of Einsteindetected bursts for six model halos with different limiting radii. No X-ray flashes were detected in the halos described by any of these six models.
Column 1 of Table 2 lists the value of $r_{l i m}$ in equation 1. Column 2 lists the limiting distance at which bursts can be detected by Einstein if the bursts at the BATSE flux limit are at a distance $r_{l i m}$. Column 3 lists the total exposure time for all galaxies with $M_{v}<-16$ whose halos fall within the field of view of an Einstein exposure. Column 4 lists the adjusted exposure time. To compute this quantity, the actual exposure time for each halo was reduced by the square of the ratio of the halo's distance to the limiting distance. Note that if galaxies were distributed uniformly in space, column 4 would always equal half of column 3. Columns 5 and 6 give the number of bursts whose detection is expected and the probability that no bursts would be detected if the model applied. Column 7 lists the probability of no bursts being detected if the physical extent of the halo were twice the distance at which BATSE is able to detect bursts.

For $r_{l i m}=600 \mathrm{kpc}$, bursts would be observable from the Virgo cluster. We would easily see them, since Einstein observed in the direction of the cluster for 4,243,000 seconds, often with multiple galaxies in the field of view. Indeed, one burst is seen in the direction of the Virgo cluster (burst \# 3 in Table 1 of Paper I). This is consistent with the expected random occurrence rate, and is inconsistent with the 23 bursts from Virgo we would see if typical halos had $600 \mathrm{kpc}$ radii. A halo this large would also produce an anisotropy in the direction of M31 observable with BATSE (Hakkila et al. 1994). Our exclusion of such models is therefore an independent confirmation of the M31 results.

We have also considered the possibility that the BATSE does not sample the entire extent of the Galactic halo. There is, of course, no reason why the halo could not extend well beyond BATSE's sampling distance. Note that BATSE's non-detection of a dipole towards M31 excludes halos larger than 400 kpc only if BATSE is able to detect halos that large. That is, BATSE obviously can not constrain the location of bursts it cannot see. However, our experiment can test for the existence of halos extended well beyond the BATSE limit. Such halos produce many more expected bursts in our galaxy sample and can be readily excluded as seen in Table 2 .

We therefore conclude that the GRBs detected by BATSE are not associated with X-ray bursts coming from a Galactic halo with a limiting radius greater than $250 \mathrm{kpc}$ and less than $400 \mathrm{kpc}$, or, equiv- 
alently, from bursts with luminosities between $7 \times$ $10^{38} \mathrm{ergs} \mathrm{s}^{-1}$ and $2 \times 10^{39} \mathrm{ergs} \mathrm{s}^{-1}$ in the $0.16-3.5 \mathrm{keV}$ band. This is the range of halo radii favored by the analysis of Hakkila et al. (1994b). Although our exclusion of halo models with limiting radii as small as $150 \mathrm{kpc}$ is only weakly significant $(73 \%)$, this result is much stronger if combined with the prior result of Hakkila et al. (1004b). If the GRBs originate in a $150 \mathrm{kpc}$ halo, then three independent probabilities must be considered: 1) this halo radius is at the $90 \%$ confidence contour of Hakkila et al.'s (1994b) result; and 2) our result excludes such a halo with $73 \%$ confidence; 3) BATSE must have been fortuitously designed to see most of the way to the halo's edge but not beyond. The a priori probability of these three independent coincidences is approximately $1 \%$. That is, combining our result with that of Hakkila et al. (1994b) excludes all halo models with $\geq 99 \%$ confidence. If we believe that X-ray counterparts are a common feature of GRBs, this would argue strongly for a cosmological GRB origin. The regions of parameter space allowed by Hakkila (1994b)'s results and ours are illustrated in Figure 1. As discussed below this chart uses the conservative and inconsistent assumption that GRBs are standard candles in both the $\gamma$-ray and X-ray bands. Deviation from either of these assumptions results in the exclusion of halo models with greater confidence.

\section{Robustness of our Conclusion}

We consider the uncertainty in the GRB X-ray / $\gamma$-ray flux to be easily the weakest link in our argument. Current models for the production of GRBs in a Galactic halo do not predict a sharp low-energy cutoff at the Einstein spectral band. Indeed a wide variety of fireball models predict a substantial X-ray excess above what we have used in our calculations (Mészáros \& Rees 1993). However, in the absence of a well-established model for the GRB production mechanism, the possibility that GRB spectra suddenly cutoff at the boundary of the Einstein and Ginga bands cannot be excluded. Unfortunately CGRO does not carry an instrument capable of measuring the spectra of the faint bursts it detects down to X-ray wavelengths. It is likely that in the next few years, however, new experiments will remedy this lack of knowledge and establish definitively the X-ray character of the GRBs.

Closely related to the uncertainty in X-ray / $\gamma$-ray flux ratio is our use of the assumption that GRBs are X-ray standard candles. This is inconsistent with the assumption of Hakkila et al. (1994a) that $\gamma$-rays from GRBs are standard candles, because the ratio of $\mathrm{X}$-ray / $\gamma$-ray flux is known to vary widely (Yoshida et al. 1989; Laros et al. 1984). Moreover, BATSE reports wide variation in the spectrum of the $\gamma$-rays it observes (Band et al. 1993). Given this spectral variability it is highly unlikely that any experiment would measure exactly a band in which the GRBs were standard candles.

In the interpretation of both the BATSE and IPC results, non-standard candles tend to reduce the parameter space available for halo models. In particular for the IPC result, the assumption of non-standard candles increases the distance at which some bursts could be detected for a given halo model. Since the volume of space from which bursts can be detected with a luminosity $L$ increases as $L^{3 / 2}$, the total number of detectable bursts increases. In the context of our models this means that bursts four times brighter than average from a, say, $200 \mathrm{kpc}$ radius model would be detected in the galaxy searches performed for the $400 \mathrm{kpc}$ radius model. Inasmuch as the volume searched in the higher radius models includes many more galaxies, non-standard candle models are excluded with higher confidence, just as are the higher radius models. For the $400 \mathrm{kpc}$ model, a factor of two excursion above average in luminosity would result in bursts visible from the Virgo cluster, a result we strongly exclude.

Another implicit assumption of our analysis is that the halos of nearby galaxies resemble that of the Milky Way. The burster halos we are searching for are at galactocentric distances far greater than the visible extent of the galaxy's light. Consequently, no kinematic evidence exists relevant to the size or frequency of such halos. Even if dark halos were shown to exist about these galaxies, there is no reason to believe that the distribution of GRB source sites would trace the mass distribution. Indeed, halo models that satisfy BATSE isotropy constraints show less source concentration toward the center of the Galaxy than halo models derived from rotation curve analysis (Hakkila et al. 1994). Because of the fast time scale of observed GRBs, it is clear they must originate from compact sources. Most models for a Galactic origin of the GRBs postulate an association with neutron stars. Recent observations of neutron star proper motions suggest that the halo may be populated with high 
velocity neutron stars that were created during the course of the star formation history of the Galaxy; i.e., they are not primordial (Lyne \& Lorimer 1994).

Support for this hypothesis follows from the recent association of supernova remnants with soft gamma repeaters (SGRs) (Murakami et al. 1994). The SGRs appear to be associated with young, high-velocity neutron stars (Rothschild, Kulkarni, \& Lingenfelter 1994). Perhaps such objects may in time populate an extended halo about any galaxy with an appropriate history of supernovae. If this is the case, it is not completely obvious what types of galaxies would have what types of halos. There may be a complex relationship between mass, galaxy type and halo extent or density. Knowing little, we have followed a simple approach. Because we make the implausible assumption that the BATSE detection limit represents an absolute limit on the burst population - i.e., that there are no bursts in our Galaxy below the BATSE limit - we consider our estimates to be conservative. However it is obviously possible that our Galaxy is anomalous with respect to its GRB source population.

T. T. H. acknowledges support from NASA grant NAGW-4110 and wishes to thank Fiona Harrison, David Hogg, and Stephen Thorsett for useful discussions. D. J. H. acknowledges support from NASA grant NAS5-32063 and gwishes to express his gratitude to his local wine merchant whose case-discount policy has allowed him to avoid bankruptcy in covering his bets that GRBs were Galactic.

\section{REFERENCES}

Band, D., et al. 1993, ApJ, 413, 281

Fich, M. \& Tremaine, S., 1991, ARA\&A, 29, 409

Fishman, et al. 1989, in Proc. Gamma-ray Observatory Science Workshop, ed. W. N. Johnson (Greenbelt: NASA), 2, 39

Gott, J. R. III \& Turner, E. L. 1976, ApJ, 209, 1

Gotthelf, E. V., Hamilton, T. T., \& Helfand, D. H. 1996, ApJ, in press (Paper I)

Hakkila, J. et al. 1994a in Proceedings of the 1993 Huntsville Gamma-Ray Burst Conference, in press

Hakkila, J., Meegan, Ca., Pendleton, G., Fishman, G., Wilson, R., Paciesas, W., Brock, M., \& Horack, J. 1994b, ApJ, 422, 659

Innanen, K. A., Harris, W. E., \& Webbink, R. F. 1983, AJ, 88, 338
Rothschild, R. E., Kulkarni, S. R., \& Lingenfelter, R. E. 1994, Nature, 368, 432

Laros, J. G. et al. 1984, ApJ, 286, 681

Lyne, A. G. \& Lorimer, D.R., 1994, Nature, 369, 127

Meegan, C. A., Fishman, G. J., Wilson, R. B., Paciesas, W. S., Pendleton, G. N., Horack, J. M., Brock, M. N., \& Kouveliotou, C. 1992, Nature, 355,143

Meegan, C. A., et al. 1994, Status report available from gronews@grossc.gsfc.nasa.gov

Meźaŕos, P. \& Rees, M. 1993, ApJ, 418, L59

Murakami, T., et al. 1991, Nature, 350, 592

Murakami, T., et al. 1994, Nature, 368, 127

Norris, J. P., Share, G. H., Messina, D. C., Dennis, B. R., desai, U. D., Cline, T. L., Matz, S. M., \& Chupp, E. L., 1986, ApJ, 301, 213

Paczyński, B. 1986, ApJ, 308, L43

Paczyński, B. \& Rhoads, J. 1993, ApJ, 418, L5

Podsiadlowski, P., Rees, M., \& Ruderman, M. A., 1995, MNRAS, 273, 755

Smith, I., A. \& Lamb, D. Q. 1993. ApJ, 410, L23

Stark, A. A., et al. 1992 ApJS, 79, 77

Tully, B. 1988, Nearby Galaxies Catalog (Cambridge: Cambridge Univ. Press)

Trimble, V. 1987 ARA\&A, 25, 425

Yoshida, A., et al. 1989, PASJ, 41, 509

This 2-column preprint was prepared with the AAS LATEX macros $\mathrm{v} 4.0$. 
TABle 1

List of Galaxies

\begin{tabular}{|c|c|c|c|c|c|c|c|}
\hline $\begin{array}{l}\text { Right Ascension } \\
\qquad(1950)\end{array}$ & $\begin{array}{l}\text { Declination } \\
\quad(1950)\end{array}$ & $\begin{array}{c}\text { Distance } \\
(\mathrm{Mpc})\end{array}$ & $\begin{array}{l}\text { Time } \\
(\mathrm{s})\end{array}$ & $\begin{array}{c}\text { Right Ascension } \\
(1950)\end{array}$ & $\begin{array}{c}\text { Declination } \\
(1950)\end{array}$ & $\begin{array}{l}\text { Distance } \\
(\mathrm{Mpc})\end{array}$ & $\begin{array}{l}\text { Time } \\
(\mathrm{s})\end{array}$ \\
\hline \multicolumn{4}{|c|}{ Galaxies at $4-12 \mathrm{Mpc}$} & 050936 & 6231 & 4.5 & 14821 \\
\hline 004318 & -1552 & 11.6 & 0 & 051006 & -3302 & 10.2 & 0 \\
\hline 004918 & 4717 & 11.8 & 0 & 051342 & 5330 & 11.4 & 0 \\
\hline 012712 & -0130 & 10.6 & 12888 & 054512 & -3415 & 10.2 & 9656 \\
\hline 013400 & 1532 & 9.7 & 6867 & 053306 & 0324 & 10.3 & 0 \\
\hline 013942 & 1343 & 10.8 & \multirow{2}{*}{$\begin{array}{l}0 \\
0\end{array}$} & 060824 & -3406 & 7.9 & 1921 \\
\hline 014018 & 1323 & 11.8 & & 032700 & 3931 & 8.6 & 0 \\
\hline 014442 & 2705 & 6.4 & 8339 & 070636 & 4432 & 8.2 & 2610 \\
\hline 014500 & 2711 & 7.5 & 8339 & 073206 & 6543 & 4.2 & 82623 \\
\hline 014642 & 3220 & 4.6 & 72619 & 073500 & -4731 & 10.9 & 0 \\
\hline 015824 & 2835 & 4.7 & 16197 & 075812 & 5054 & 10.1 & 0 \\
\hline 021918 & 4207 & 9.6 & 10364 & 080942 & 4609 & 9.0 & 8390 \\
\hline 022154 & 3549 & 9.8 & 6335 & 081024 & 4554 & 10.6 & 0 \\
\hline 022418 & 3322 & 9.4 & 10474 & 081100 & 4913 & 10.6 & 8390 \\
\hline 022748 & 3655 & 10.3 & 0 & 081406 & 7052 & 4.5 & 57834 \\
\hline $02 \quad 2918$ & 3517 & 10.1 & 6335 & 081542 & 5010 & 10.0 & 8390 \\
\hline 023018 & 3317 & 10.1 & 6335 & 084936 & 3338 & 5.7 & 13479 \\
\hline 023036 & 4019 & 10.2 & 2748 & 085548 & 3924 & 8.7 & 4249 \\
\hline 023324 & 2513 & 10.7 & 1432 & 090424 & 3328 & 7.8 & 1319 \\
\hline 023606 & 4040 & 10.7 & 2748 & 091006 & -2358 & 7.1 & 6102 \\
\hline 023718 & 3851 & 10.5 & 2748 & 091542 & -2209 & 10.8 & 6102 \\
\hline 023742 & 1905 & 11.2 & 1972 & 091836 & 5112 & 12.0 & 0 \\
\hline 024012 & 3708 & 9.1 & 1629 & 092924 & 2144 & 6.3 & 11298 \\
\hline 024448 & 3720 & 10.0 & 1629 & 095142 & 6955 & 5.2 & 37258 \\
\hline 025524 & -5446 & 5.4 & 5900 & 100054 & 4100 & 9.4 & 0 \\
\hline 025648 & 2502 & 6.4 & 9961 & 100242 & - 0729 & 6.7 & 4111 \\
\hline 030836 & -5332 & 10.7 & 5900 & 101512 & 4140 & 8.7 & 614 \\
\hline 031530 & -4119 & 8.6 & 2809 & 101642 & 4549 & 10.8 & 0 \\
\hline 032418 & -5257 & 11.5 & 10890 & 102224 & 1725 & 8.1 & 8496 \\
\hline 033012 & -5205 & 11.6 & 6436 & 103624 & 4156 & 11.5 & 0 \\
\hline 033154 & -3122 & 11.6 & 562 & 104048 & 2511 & 6.1 & 0 \\
\hline 033706 & -4415 & 11.2 & 2016 & 104118 & 1158 & 8.1 & 29026 \\
\hline 033718 & -1851 & 5.0 & 26936 & 104342 & 0205 & 10.7 & 2576 \\
\hline 033730 & -3130 & 11.8 & 1646 & 104412 & 1205 & 8.1 & 29026 \\
\hline 034030 & -4723 & 11.6 & 0 & 104506 & 1415 & 8.1 & 20724 \\
\hline 035554 & -4621 & 11.3 & 2016 & 104512 & 1251 & 8.1 & 20724 \\
\hline 040154 & -0219 & 10.6 & 915 & 104536 & 1254 & 8.1 & 26872 \\
\hline 040154 & -4333 & 10.3 & 2052 & 104818 & 1341 & 8.1 & 28961 \\
\hline 040218 & -4329 & 9.5 & 2052 & 104800 & 7607 & 10.9 & 3767 \\
\hline 040654 & -4801 & 11.0 & 0 & 104942 & 3654 & 7.8 & 9728 \\
\hline 043854 & -0256 & 8.9 & 5975 & 105742 & 1410 & 6.4 & 42074 \\
\hline 045306 & -5327 & 6.0 & 14185 & 105748 & 2915 & 7.4 & 14465 \\
\hline 045754 & -2606 & 7.8 & 1448 & 110100 & 2909 & 7.9 & 16049 \\
\hline 050206 & -6112 & 10.6 & 0 & 110312 & 0014 & 7.2 & 0 \\
\hline 050430 & -3201 & 7.4 & 0 & $0 \quad 111736$ & 1317 & 6.6 & 20902 \\
\hline 050600 & -3735 & 10.8 & 0 & $\begin{array}{lll}9 & 11 & 1742\end{array}$ & 1353 & 7.7 & 20902 \\
\hline 050848 & -3140 & 10.8 & 0 & 113300 & 5447 & 4.3 & 39312 \\
\hline & & 10.0 & & 115406 & 4836 & 8.3 & 4617 \\
\hline
\end{tabular}


Table 1 (Continued)

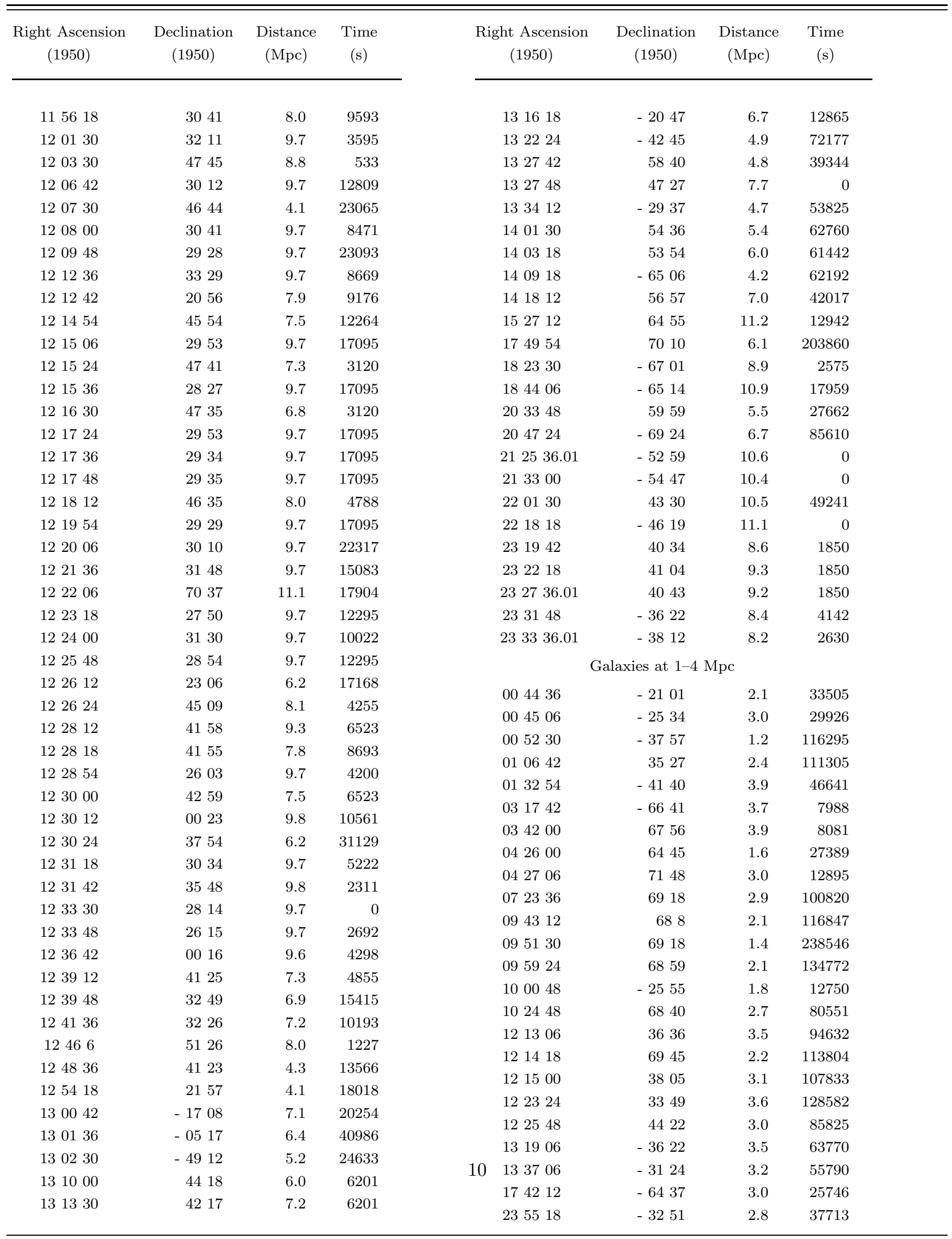


TABle 2

Halo Models

\begin{tabular}{|c|c|c|c|c|c|c|}
\hline $\begin{array}{l}r_{l i m} \\
(\mathrm{kpc})\end{array}$ & $\begin{array}{l}D_{\text {lim }} \\
(\mathrm{Mpc})\end{array}$ & $\begin{array}{l}\text { Total Time } \\
\quad(\mathrm{ksec})\end{array}$ & $\begin{array}{c}\text { Adjusted Time } \\
(\mathrm{ksec})\end{array}$ & $\begin{array}{c}\text { Expected } \\
\text { Events }\end{array}$ & Significance & $\begin{array}{c}\text { Probability of } \\
\text { Doubled Radius }\end{array}$ \\
\hline 150 & 4.5 & 391 & 208 & 1.4 & .239 & 0.027 \\
\hline 200 & 6.0 & 638 & 292 & 2.0 & .135 & 0.004 \\
\hline 250 & 7.5 & 1020 & 470 & 3.2 & .041 & $<0.0001$ \\
\hline 300 & 9.0 & 1290 & 606 & 4.2 & .015 & $<0.0001$ \\
\hline 350 & 10.5 & 1590 & 829 & 5.7 & .003 & $<0.0001$ \\
\hline 400 & 12.0 & 1890 & 915 & 6.3 & .002 & $<0.0001$ \\
\hline
\end{tabular}




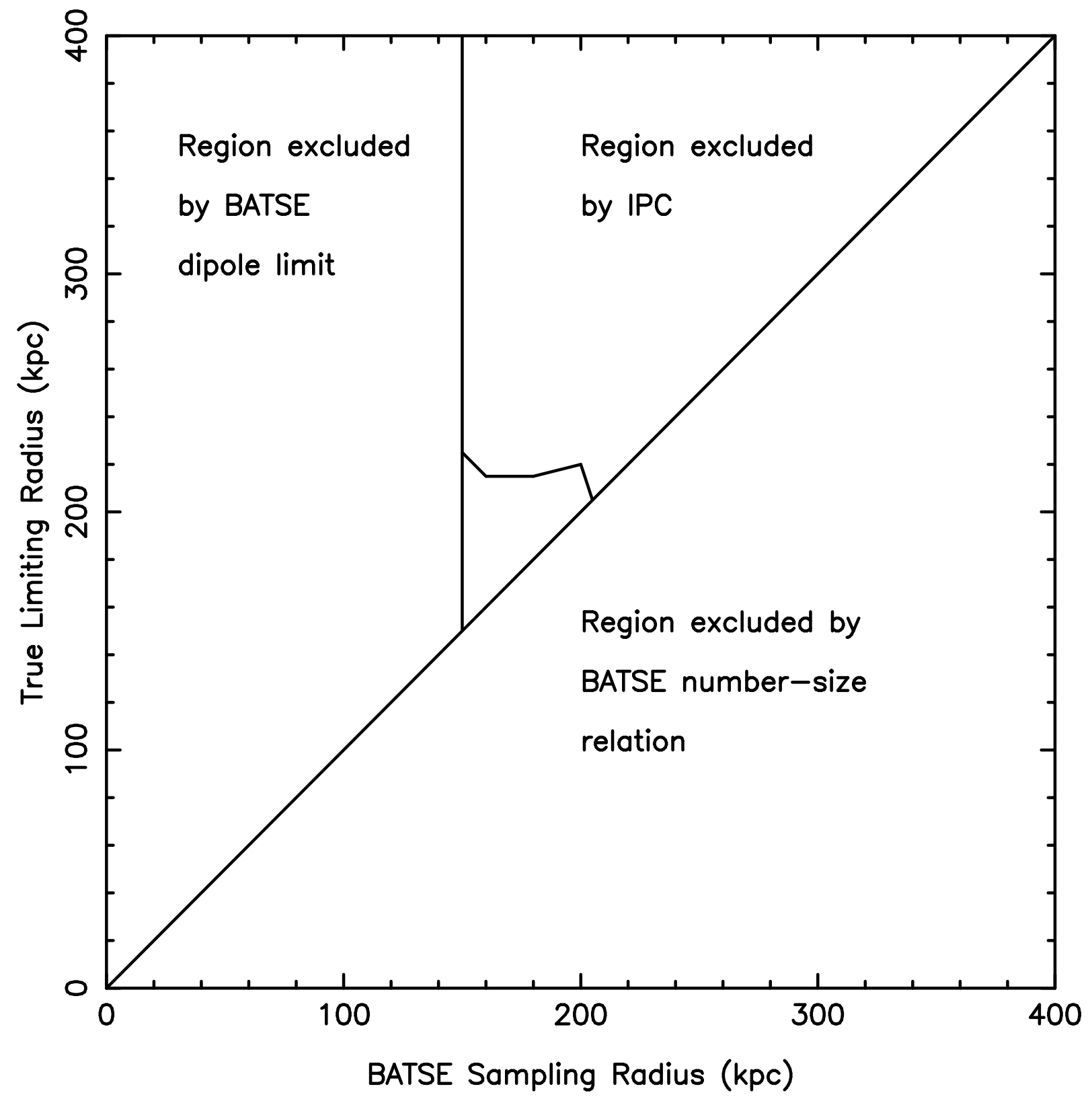

Fig. 1. - The region of parameter space of possible halo models excluded with $>90 \%$ confidence by various experiment is plotted. The abscissa is the distance of the faintest bursts detected by BATSE and the ordinate is the distance of the faintest bursts which exist. We have assumed standard candles and a continuation of the Log $N-\log S$ below the BATSE limit with the same slope. We allow the inner radius of the distribution to assume any value. For BATSE models in the area not excluded by any one experiment, that radius is about $20 \mathrm{kpc}$. The irregular shape of the IPC contour is a result of the finite number of nearby galaxies. 